02,13

\title{
Изучение эволюции атомного состава тонких пленок NbN в процессе облучения смешанными ионными пучками методами спектроскопии энергетических потерь электронов
}

\author{
(С) М.М. Дементьева ${ }^{1}$, К.Е. Приходько ${ }^{1,2}$, Б.А. Гурович ${ }^{1}$, Л.В. Кутузов ${ }^{1}$, Д.А. Комаров ${ }^{1}$ \\ ${ }^{1}$ НИЦ „Курчатовский институт“, \\ Москва, Россия \\ ${ }^{2}$ Национальный исследовательский ядерный университет (МИФИ), \\ Москва, Россия \\ E-mail: dementyeva_mm@nrcki.ru
}

\begin{abstract}
Экспериментально методами спектроскопии энергетических потерь электронов в просвечивающем электронном микроскопе в режиме трансмиссионного сканирования на образцах поперечных срезов исследовано изменение атомного состава ультратонких пленок $\mathrm{NbN}$ в ходе их облучения смешанными ионными пучками до дозы $4 \mathrm{dpa} \mathrm{(по} \mathrm{азоту).} \mathrm{Установлено} \mathrm{протекание} \mathrm{процесса} \mathrm{замещения} \mathrm{атомов} \mathrm{азота} \mathrm{атомами} \mathrm{кислорода,}$ характеризующееся изменением состава проводящей части пленки с $\mathrm{NbN}$ на $\mathrm{NbNO}$.
\end{abstract}

Работа поддержана Министерством образования и науки РФ (соглашение о предоставлении субсидии № 14.607.21.0005, уникальный индентификатор RFMEFI60714X0005).

\section{1. Введение}

Система $\mathrm{NbN}$ с типом кристаллической решетки $B 1$ (или $\mathrm{NaCl}$ ), обладающая сверхпроводящими свойствами, высоким значением критического тока и самой высокой критической температурой сверхпроводящего перехода $T_{c}=12-14 \mathrm{~K}$ (в случае ультратонких пленок) среди сверхпроводников типа $B 1[1]$, является перспективной для создания функциональной элементной базы криоэлектроники. К основным устройствам криоэлектроники относятся сверхпроводящие провода, на основе которых создаются активные (сверхпроводящие однофотонные счетчики, болометры на горячих электронах) и пассивные (конденсаторы, резисторы, индуктивности и приборы, работа которых основана на эффекте Джозефсона, например сверхпроводящий квантовый интерференционный прибор (СКВИД)) элементы наноразмерных масштабов. Для широкого внедрения таких устройств существует необходимость разработки технологии их изготовления, обеспечивающей высокое качество таких приборов. Разработанный в НИЦ „Курчатовский институт“ комплекс радиационных методов управляемой модификации химического состава и свойств тонкопленочных материалов в процессе облучения низкоэнергетическими ионными пучками [2] позволяет создавать композитные материалы для криоэлектронных приложений.

Основа методов радиационно-индуцированной трансформации свойств веществ, например, при создании материала, проявляющего металлические свойства при криогенных температурах, заключается в модификации атомного состава тонкопленочного сверхпроводника за счет воздействия на выбранные участки вещества потоками ускоренных частиц смешанного состава: ионов водорода с энергией, достаточной для создания радиационных повреждений в исходном веществе, и ионов, которые требуется ввести в состав формируемого хи- мического соединения, - так называемый метод селективного замещения атомов. В результате такого облучения инициируется процесс замены атомов исходного химического соединения на новые атомы, содержащиеся в ионном пучке (в данном случае замена атомов азота в нитриде ниобия на атомы кислорода).

При создании функциональных элементов радиационными методами необходимо установить степень модификации исходной пленки и экспериментально изучить ее состав для полноценного сопоставления с измеряемыми электрофизическими свойствами. Ранее в ряде работ [3-5] также изучалось влияние радиационного облучения на сверхпроводящие свойства нитрида ниобия. Было показано, что облучение быстрыми нейтронами до флюенса $1.5 \cdot 10^{20}$ neutrons $/ \mathrm{cm}^{2}$ приводит к понижению температуры сверхпроводящего перехода всего на 6\% [3]. Такая высокая устойчивость к радиационному повреждению объясняется дефектной природой пленок $\mathrm{NbN}$ [3]. В то же время облучение пленок высокоэнергетическими ионами благородных газов $\mathrm{Ar}^{+}$ с энергией $200 \mathrm{keV}$ в работе [4] и ионами $\mathrm{Ne}^{+}$с энергией $350 \mathrm{keV}$ в работе [5] приводит к значительному снижению температуры перехода из-за возникновения радиационных повреждений. Экспериментально было установлено, что изменение значения электрического сопротивления, происходящее вследствие появления радиационно-индуцированных повреждений, выходит на насыщение при дозе, большей $1 \mathrm{dpa}$ (dpa - displacement per atom) [5]. Проведенные эксперименты по изучению влияния облучения благородными газами больших энергий $[4,5]$ информативны с точки зрения исследования влияний радиационных дефектов на электрические свойства массивных сверхпроводников. Однако в случае использования тонкопленочных сверхпроводников существует ряд ограничений на значения энергий ионов и их тип в связи с эффектом физического распыления поверхности. 
В настоящей работе в отличие от перечисленных выше публикаций объектом исследований являются ультратонкие пленки $\mathrm{NbN}$, толщина которых составляет $5 \mathrm{~nm}$. В связи с этим используются смешанные ионные пучки на основе протонов с низкой энергией для изучения влияния облучения на протекание радиационно-индуцированного процесса замещения атомов азота атомами кислорода.

С учетом малой толщины пленки (несколько нанометров) требуется использование методов просвечивающей электронной микроскопии в режиме трансмиссионного сканирования, поскольку в этом случае локальность получения аналитической информации о составе материала определяется размером сфокусированного электронного зонда и составляет $\sim 0.14 \mathrm{~nm}$. Анализ элементного состава по глубине пленки может быть выполнен на образцах поперечных срезов методом спектроскопии характеристических потерь энергии электронов (СХПЭЭ), основанным на анализе спектров неупругорассеянных электронов и позволяющим получать количественные данные о концентрациях легких элементов (азот, кислород), а также ниобия.

\section{2. Эксперимент}

Образцы тонких сверхпроводящих пленок нитрида ниобия толщиной $\sim 5 \mathrm{~nm}$, нанесенные на монокристаллическую подложку кремния, покрытую термическим оксидом $\mathrm{SiO}_{2}$, изготавливались методом катодного распыления ниобия в контролируемой газовой среде азота и криптона при давлении $10^{-5}$ Torr и температуре нагрева подложки $\sim 800^{\circ} \mathrm{C}[6]$.

Изменение атомного состава пленок нитрида ниобия проводилось в процессе облучения смешанными низкоэнергетическими ионными пучками, состоящими из ионов кислорода и протонов. Поскольку цель настоящей работы - создание на основе сверхпроводника пассивного элемента электрической цепи с металлическим типом проводимости, состав пучка определялся так, чтобы в процессе модификации состава исходной пленки $\mathrm{NbN}$ лишь часть атомов азота заместилась атомами кислорода и глубина изменения соответствовала длине проективного пробега протонов. Энергии ионов составляли $0.1-1 \mathrm{keV}$, ионный пучок состоял из протонов и ионов ОН, его состав [2] соответствовал $c=p(\mathrm{OH}) / p\left(\mathrm{H}_{2}+\mathrm{OH}\right)=1.2 \cdot 10^{-3}$ (состав ионного пучка контролируется как отношение парциального давления ионов ОН к суммарному давлению водорода и ионов $\mathrm{OH}$ в процессе эксперимета). Скорость набора дозы по азоту составляла $\sim 0.02 \mathrm{dpa} / \mathrm{s}$, облучение проводилось в диапазоне доз по азоту от 1.6 до $4 \mathrm{dpa}$.

Анализ структуры и химического состава пленок проводился с использованием просвечивающего электронного микроскопа „Titan 80-300ST“(при ускоряющем напряжении $200 \mathrm{kV}$ ), оснащенного спектрометром для регистрации спектров энергетических потерь электронов.

Изготовление образцов поперечных срезов $\mathrm{NbN} / \mathrm{SiO}_{2} / \mathrm{Si}$ толщиной $\sim 20-50 \mathrm{~nm}$ проводилось на установке с фокусированным ионным пучком (FIB) „Helios Nanolab 650“ при ускоряющем напряжении ионной пушки $30 \mathrm{kV}$ и токе $2.5 \mathrm{nA}$ и окончательном утонении при напряжении $2-5 \mathrm{kV}$ и токе $120 \mathrm{pA}$.

Для количественного анализа распределения элементов по глубине облученных пленок при различных дозах использовался метод СХПЭЭ в режиме просвечивающей растровой электронной микроскопии (ПРЭМ). Спектры потерь электронов детектировались с помощью спектрометра GIF-2001. Методы СХПЭЭ, применяемые на образцах поперечных срезов, позволили получить профили распределения элементов по всей глубине пленки методом относительных концентраций

$$
\frac{N_{A}}{N_{B}}=\frac{I_{A}(\beta, \Delta)}{I_{B}(\beta, \Delta)} \frac{\sigma_{B}(\beta, \Delta)}{\sigma_{A}(\beta, \Delta)},
$$

где $I_{A}, I_{B}-$ интегральные интенсивности пиков под кривыми поглощения после вычитания фона, $\sigma_{A}$ и $\sigma_{B}$ сечения процессов неупругого рассеяния атомов $A$ и $B$ для данного угла сбора спектрометра $\beta$ [7], $\Delta-$ диапазон энергий для расчета интегральных интенсивностей пиков. В (1) $N_{A} / N_{B}$ - отношение числа атомов элемента $A$ к числу атомов элемента $B$. В случае многоатомных соединений экспериментально определяются данные соотношения для различных элементов соединения и пересчитываются в относительные атомные концентрации элементов.

\section{3. Результаты и обсуждение}

В настоящее время известно [6], что облучение пленок нитрида ниобия смешанными ионными пучками приводит к эволюции их электрофизических свойств. Как было показано в [8], при облучении нитрида ниобия смешанными ионными пучками на дозовой зависимости значения сопротивления на квадрат при температуpe $4.3 \mathrm{~K}$ для облученных пленок наблюдается плавный

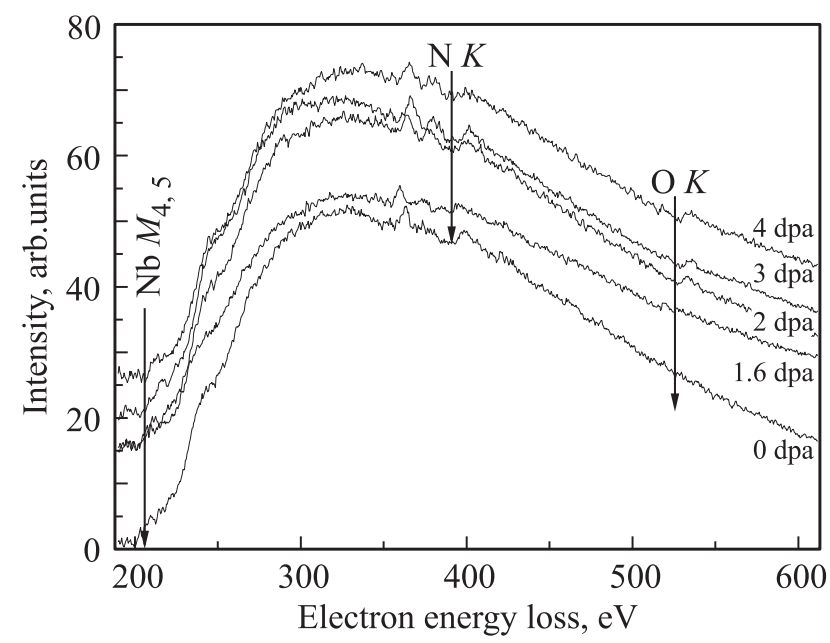

Рис. 1. Характеристические спектры энергетических потерь электронов для пленки $\mathrm{NbN}$ в диапазоне доз облучения $0-4$ dpa. 



Рис. 2. Профили распределения элементов по глубине пленки $\mathrm{NbN}$ (от поверхности образца), построенные методом анализа спектров характеристических потерь энергии электронов, измеренных в режиме ПРЭМ, при дозах облучения $0(a), 1.6(b), 2(c)$, $3(d)$ и 4 dpa $(e)$.

подъем от $\sim 800$ до $\sim 2000 \Omega / \square$ в диапазоне доз от $\sim 2$ до $\sim 9 \mathrm{dpa} \mathrm{(по} \mathrm{азоту)} \mathrm{при} \mathrm{сохранении} \mathrm{металлического}$ характера проводимости пленки.

Целью настоящей работы является изучение эволюции элементного состава по глубине тонкой пленки нитрида ниобия в результате воздействия смешанного ионного облучения аналитическими методами просвечивающей электронной микроскопии. Далее приведе- ны результаты анализа изменения элементного состава по глубине пленок, облученных при различных дозах по азоту.

На рис. 1 представлены типичные спектры характеристических энергетических потерь электронов для исходного образца и образцов, облученных при различных дозах по азоту. Как видно из этих спектров, при повышении дозы облучения растет максимум края 
поглощения кислорода $(532 \mathrm{eV})$, обусловленный возбуждением электронов внутренней $K$-оболочки.

На рис. 2 приведены профили распределения элементов по глубине исходной $(a)$ и облученных $(b-e)$ пленок. Точками на рис. 2 обозначены экспериментальные значения относительных концентраций элементов, рассчитанные из спектров характеристических потерь электронов, а линиями показаны их интерполяционные зависимости. Из вида распределений элементов по глубине пленки очевидно, что ее можно рассматривать как состоящую из двух частей: верхней части пленки, прилегающей к облучаемой поверхности образца, и нижней части пленки, прилегающей к подложке из оксида кремния.

Как видно из рис. $2, a$, в исходной пленке верхняя часть обогащена кислородом, а в нижней части пленки концентрация элементов близка к стехиометрическому составу NbN. Между верхней и нижней частями пленки находится переходная область, центр которой можно условно рассматривать в качестве границы, разделяющей верхнюю и нижнюю части пленки. Дальнейший анализ эволюции состава пленок при различных дозах облучения представлен в терминах измерений средних концентраций отдельных элементов в верхней и нижней частях пленки, а также в центре переходной зоны между верхней и нижней частями пленки. Под центром переходной зоны понимается такая глубина, на которой концентрация элементов соответствует среднему арифметическому между концентрациями в верхней и нижней частях пленки. В большинстве случаев центры переходной зоны, рассчитанные по разным элементам, соответствуют друг другу.

Из анализа спектров на рис. 2, во-первых, следует, что с увеличением дозы смешанного ионного облучения в верхней части пленки концентрация атомов азота падает до нуля, а соотношение концентраций атомов кислорода и ниобия соответствует окисленному состоянию ниобия. Во-вторых, видно, что толщина верхнего окисленного слоя с ростом дозы облучения увеличивается. Это обусловлено объемными изменениями, сопровождающими образование оксида ниобия из нитрида ниобия. Втретьих, видно, что с ростом дозы облучения в нижней части пленки концентрация атомов азота уменьшается, но не падает до нуля, а концентрация атомов кислорода увеличивается, т.е. имеет место частичное замещение атомов азота атомами кислорода вследствие протекания процесса селективного замещения атомов.

Поскольку проводящими свойствами обладает именно нижняя часть пленки, в которой произошло частичное замещение атомов азота атомами кислорода, на рис. $3, a$ представлены зависимости средних концентраций элементов в нижней части пленки от дозы облучения. Как следует из этого рисунка, при дозах облучения более двух смещений на атом (по азоту) средняя концентрация трех основных элементов в нижней части пленки выравнивается, что соответствует проведенному ранее фазовому анализу структуры облученной пленки,
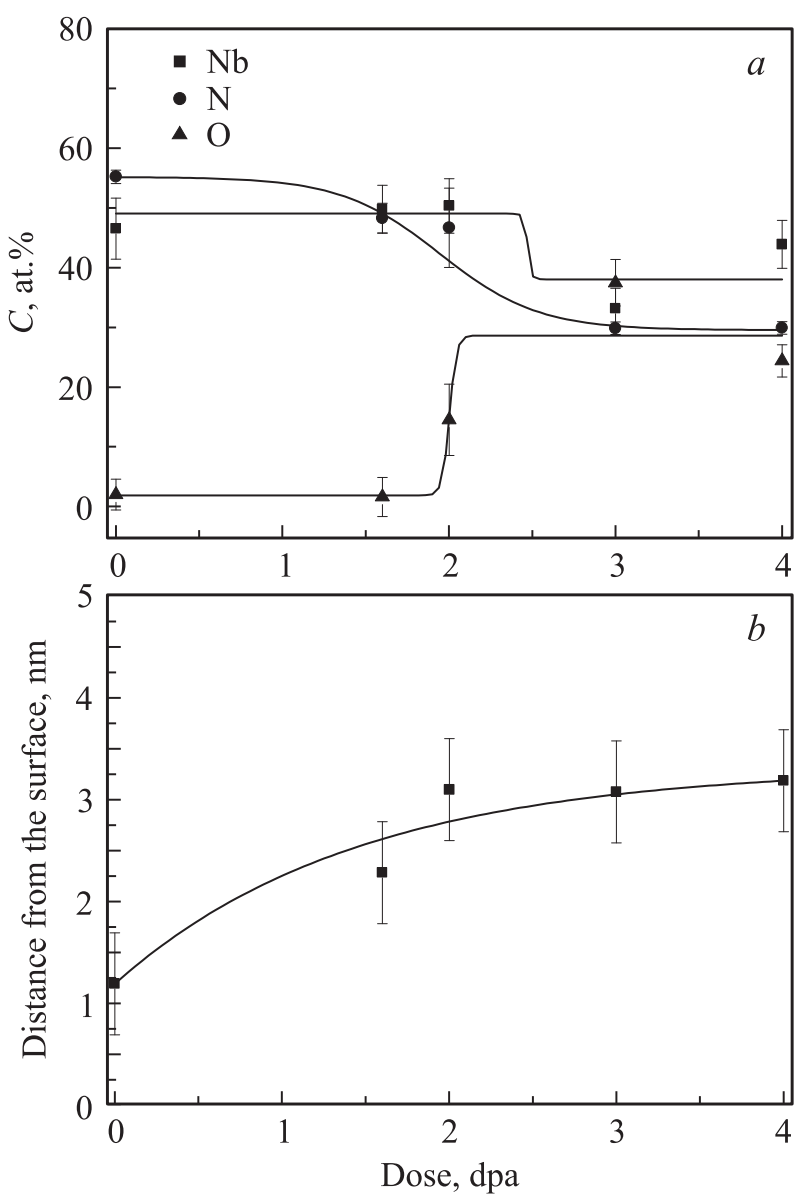

Рис. 3. Зависимость значений концентраций элементов в области пленки, прилегающей к подложке $(a)$, а также значения толщины верхнего окисленного слоя $(b)$ от дозы смешанного облучения.

показавшему образование отдельных зерен, близких к составу NbNO [9]. При этом с увеличением дозы облучения растет толщина верхнего окисленного слоя, выходя при этом на насыщение (рис. $3, b$ ).

\section{4. Заключение}

В работе экспериментально показано, как изменяется атомная концентрация элементов по толщине тонкой пленки нитрида ниобия в процессе ее облучения смешанным ионным пучком в диапазоне доз по азоту до $4 \mathrm{dpa}$. Показано, что под воздействием смешанного облучения протекает процесс замещения атомов азота атомами кислорода, при этом внешний слой пленки окисляется, а внутренние слои пленки отвечают среднему составу NbNO. Контролируемое изменение атомного состава тонких пленок $\mathrm{NbN}$ под действием ионного облучения может быть использовано для создания функциональных криоэлектронных элементов.

Авторы выражают благодарность В.Л. Столярову и Е.Д. Ольшанскому за изготовление исходных тонких пленок нитрида ниобия. 


\section{Список литературы}

[1] T.P. Orlando, K.A. Delin. Foundation of applied superconductivity. Prentice Hall (1991). 584 p.

[2] Б.А. Гурович, К.Е. Приходько, Е.А. Кулешова. ЖЭТФ 143, 6, 1 (2013).

[3] D. Dew-Hughes, R. Jones. Appl. Phys. Lett. 36, 856 (1980).

[4] J.Y. Juang, D.A. Rudman, J. Talvacchio, R.B. van Dover. Phys. Rev. B 38, 4, 2354 (1988).

[5] Th. Siebold, P. Ziemann. Phys. Rev. B 51, 10, 6328 (1995).

[6] Б.А. Гурович, М.А. Тархов, К.Е. Приходько, Е.А. Кулешова, Д.А. Комаров, В.Л. Столяров, Е.Д. Ольшанский, Б.В. Гончаров, Д.А. Гончарова, Л.В. Кутузов. А.Г. Домантовский. Рос. нанотехнологии 9, 7-8, 36 (2014).

[7] D. Williams, A. Carter. Electron microscopy. Springer, N.Y. (2009). 775 p.

[8] B.A. Gurovich, K.E. Prikhodko, M.A. Tarkhov, A.G. Domantovsky, D.A. Komarov, B.V. Goncharov, E.A. Kuleshova. Micro and Nanosystems 8, 1 (2015).

[9] K. Prikhodko, B. Gurovich, M. Dement'eva. In: Proc. of the 10th Int. Conf. on materials for extreme conditions: development, creation, application. IOP Conf. Ser.: Mater. Sci. Eng. 130, 012046 (2016). 